\title{
Colaboración farmacéutico-médico en la elaboración de protocolos consensuados para el tratamiento de síntomas menores: programa 'INDICA+PRO'
}

\author{
Noelia Amador-Fernández ${ }^{1,2}$, Shalom I. Benrimoj3 ${ }^{3}$ Vicente J. Baixauli Fernández ${ }^{1}, M^{a}$ Teresa Climent Catalá1, \\ Vicente Colomer Molina ${ }^{4}$, Óscar Esteban Jiménez ${ }^{5}$ Buenaventura Fernández San José5, Óscar García Agudo ${ }^{4}$ \\ Victoria García Cárdenas ${ }^{3}$, José Ignacio García Garcia ${ }^{6}$, Miguel Ángel Gastelurrutia ${ }^{2}$, \\ Jesús C .Gómez Martínez ${ }^{1}$, Francisco Valls Roca ${ }^{6}$, Fernando Martínez Martínez ${ }^{2}$
}

1. Sociedad Española de Farmacia Familiar y Comunitaria (SEFAC). 2. Grupo de Investigación en Atención Farmacéutica (GIAF-UGR). 3. Graduate School of Health, University of Technology Sydney (UTS). 4. Muy Ilustre Colegio Oficial de Farmacéuticos de Valencia (MICOF). 5. Sociedad Española de Medicina de Familia y Comunitaria (SemFyC). 6. Sociedad Española de Médicos de Atención Primaria (Semergen).

\section{PALABRAS CLAVE}

Síntomas menores, protocolos, farmacia comunitaria, atención farmacéutica, servicios profesionales farmacéuticos asistenciales, atención primaria

\section{ABREVIATURAS}

Foro AF-FC: Foro de Atención Farmacéutica en Farmacia Comunitaria

GIAF-UGR: Grupo de Investigación en Atención Farmacéutica de la Universidad de Granada MICOF: Muy Ilustre Colegio de Farmacéuticos de Valencia SEFAC: Sociedad Española de Farmacia Familiar y Comunitaria SemFYC: Sociedad Española de Medicina de Familia y Comunitaria SEMERGEN: Sociedad Española de Médicos de Atención Primaria SIF: Servicio de Indicación Farmacéutica

SPFA: Servicios Profesionales Farmacéuticos Asistenciales

\section{RESUMEN}

Introducción: la colaboración entre médicos y farmacéuticos es necesaria en el manejo de los síntomas menores, ya que el paciente acude a ambos profesionales sanitarios para su tratamiento. El objetivo del estudio fue elaborar protocolos de trabajo consensuados entre farmacéuticos comunitarios y médicos de atención primaria para el Servicio de Indicación Farmacéutica (SIF).

Material y método: se utilizaron dos metodologías: revisión bibliográfica y grupo de expertos para el consenso. Estos protocolos se actualizaron y diseñaron a partir de un documento previo de 2008. En el grupo de expertos se incluyeron cuatro farmacéuticos comunitarios de SEFAC (2), MICOF (2) y cuatro médicos de atención primaria de semFYC (2) y SEMERGEN (2).

Resultados: se consensuaron protocolos de trabajo para 31 síntomas menores distribuidos en cinco grupos: respiratorios, dolor moderado, digestivos, dermatológicos y otros. Se añadieron cinco síntomas nuevos al documento de partida.

Respecto a la estructura de cada síntoma se consideró: concepto, causas más frecuentes, criterios de derivación al médico (edad, síntomas de alarma, duración de los síntomas, otros problemas de salud y/o medicamentos y situaciones especiales), recomendaciones para la prevención y tratamiento (no farmacológico y farmacológico).

Conclusión: el documento desarrollado mediante colaboración entre profesionales de la medicina y la farmacia es una herramienta que contribuye a la mejora de la actuación del farmacéutico en el manejo de los síntomas menores desde la farmacia comunitaria, con énfasis en la definición de indicadores que señalan la necesidad de derivar el paciente al médico de atención primaria.

Collaborative care protocols for the management of minor ailments agreed between pharmacists and medical practitioners: INDICA+PRO Study

\section{ABSTRACT}

Background: To increase the collaboration between community pharmacists and medical practitioners working in primary care it is critical to have collaborative protocols in place. Minor ailments could be a good example where this collaboration would benefit patient care as patients are seeking treatment in both settings. These collaborative protocols would assist in diminishing the variability of treatments in both settings. The aim of the study was to develop care protocols for minor ailments agreed between medical practitioners and pharmacists.
Financiación: el proyecto INDICA+PRO fue financiado por la Sociedad Española de Farmacia Familiar y Comunitaria (SEFAC) y el Muy Ilustre Colegio Oficial de Farmacéuticos de Valencia (MICOF). Ninguna de las organizaciones influyó en el diseño del estudio, la interpretación de los datos, la escritura del manuscrito o la decisión de enviarlo a publicación. Conflicto de intereses: ninguno.

Contribución a la autoría: los autores contribuyeron por igual al diseño del estudio, desarrollo de la investigación, análisis de los resultados, redacción del manuscrito y revisión del contenido final.

Cite este artículo como: Amador-Fernández N, Benrimoj SI, Baixauli VJ , Climent MT, Colomer V, Esteban Ó, Fernández B, Garcia O, Garcia V, Garcia Jl, Gastelurrutia MÁ, Gómez JC, Valls F, Martínez F. Colaboración farmacéutico-médico en la elaboración de protocolos consensuados para el tratamiento de sintomas menores: programa 'INDICA+PRO'. Farmacéuticos Comunitarios. 2019 Dec 30;11(4):21-31. doi:10.5672/FC.2173-9218.(2019/Vol11).004.03

Correspondencia: Noelia Amador Fernández (noelia.af@outlook.com).

ISSN 1885-8619 @SEFAC (Sociedad Española de Farmacia Familiar y Comunitaria). Todos los derechos reservados. 


\section{KEYWORDS}

Minor ailments, clinical protocols, community Pharmacy, pharmaceutical services, primary health care
Methods: Two different methodologies were used to arrive at a consensus for the protocols: literature review and expert group. Some of the clinical protocols were updated from a previous document created in 2008. Expert group was composed of four community pharmacists (SEFAC, MICOF) and four medical practitioners (SemFyC y Semergen).

Results: Thirty-one protocols for minor ailments were agreed and allocated to five groups (five new minor ailments protocols were added: respiratory tract-related conditions, gastrointestinal disturbance, pain, dermatological problems and others.

Protocols were structured following: definition, more frequent causes, referral criteria (patient's age, red flags, symptom's duration, patient's health problems and treatments and physiological situations), recommendations for management (prevention, non-pharmacological and pharmacological treatment) and references used.

Conclusions: Clinical protocols agreed between health professionals of medicine and pharmacy are a tool that contribute to better management of minor ailments in community pharmacy.

\section{Introducción}

Los síntomas menores se definen como "problemas de salud de carácter no grave, autolimitados, de corta duración, que no tienen relación alguna con las manifestaciones clínicas de los otros problemas de salud que sufra el paciente, ni con los efectos, deseados o no, de los medicamentos que toma, que no precisan por tanto diagnóstico médico y que responden o se alivian con un tratamiento sintomático" (1). El autocuidado y la automedicación son normalmente los tratamientos de elección en el manejo de los síntomas menores. La promoción de este autocuidado permite mejorar el conocimiento y las habilidades de los usuarios optimizando la toma de decisiones relacionadas con la salud. Los profesionales sanitarios tienen un papel fundamental en dicha promoción y, entre ellos, se encuentra el farmacéutico comunitario, cuyo papel ha ido evolucionando hacia una labor más asistencial a través de servicios profesionales farmacéuticos asistenciales (SPFA), definidos por Foro de Atención Farmacéutica en Farmacia Comunitaria (Foro AFFC) (2) como "aquellas actividades sanitarias prestadas desde la farmacia comunitaria por un farmacéutico que emplea sus competencias profesionales para la prevención de la enfermedad y la mejora, tanto de la salud de la población como la de los destinatarios de los medicamentos y productos sanitarios, desempeñando un papel activo en la optimización del proceso de uso y de los resultados de los tratamientos. Dichas actividades, alineadas con los objetivos generales del sistema sanitario, tienen entidad propia, con definición, fines, procedimientos y sistemas de documentación, que permiten su evaluación y retribución, garantizando su universalidad, continuidad y sostenibilidad".

En España, los síntomas menores pueden ser tratados en la farmacia comunitaria a través del Servicio de Indicación Farmacéutica (SIF) que fue definido por Foro AF-FC como "el SPFA prestado ante la consulta de un problema de salud concreto, para un paciente o cuidador que llega a la farmacia solicitando el remedio más adecuado para el mismo" (3) y clasificado dentro de los servicios de atención farmacéutica orientados al proceso de uso de los medicamentos. El procedimiento para el mismo se incluye en la figura 1.

Además, Foro AF-FC considera que aquellos casos de automedicación ("selección y uso de medicamentos por los individuos para tratar problemas de salud o síntomas autodiagnosticados" (4)) en los que se solicite un medicamento sin prescripción se podrán evaluar utilizando el procedimiento del SIF. Esta vía permite así la conversión de esa automedicación en automedicación responsable.

En la práctica habitual existe variabilidad entre farmacias comunitarias en la prestación del SIF. Por ello, es necesario disponer de criterios científicos y técnicos consensuados entre médicos y farmacéuticos que se apliquen de forma sistemática en este servicio. De manera que se potencien las contribuciones de ambos profesionales sanitarios en la mejor atención posible para las situaciones en la que un paciente se presenta en la farmacia con un sintoma menor.

El objetivo del presente estudio consistió en elaborar protocolos normalizados de trabajo consensuados entre farmacéuticos comunitarios y médicos de atención primaria para el SIF. Este estudio consistió en un fase previa del programa INDICA $+P R O$
(5), que se llevó a cabo para evaluar el impacto clínico, humanístico y económico de un SIF protocolizado en pacientes que acuden a la farmacia comunitaria para una consulta sobre los sintomas menores incluidos (digestivos: acidez, diarrea aguda, flatulencia, vómitos; relacionados con el dolor moderado: cefalea, dolor de garganta, dismenorrea; dermatológicos: herpes labial, pie de atleta; y respiratorios: congestión nasal, sindrome gripal/catarral, tos) o demandando un medicamento para los mismos, comparado con la atención habitual en farmacia comunitaria y centros de atención primaria.

\section{Material y métodos}

Diseño del estudio

Para la elaboración de los protocolos se utilizaron dos metodologías distintas: revisión bibliográfica $\mathrm{y}$ grupo de expertos para la consecución de un consenso. Estos protocolos se actualizaron y diseñaron a partir de un documento previo de 2008 (6) dentro de una fase previa de codiseño del SIF en el programa INDICA $+P R O$ con médicos de atención primaria, farmacéuticos comunitarios, organizaciones de pacientes y representantes de la administración local.

El programa INDICA $+P R O$ (5), a su vez, consistió en un ensayo controlado aleatorizado por conglomerados realizado durante seis meses desde diciembre de 2017 hasta mayo de 2018.

\section{Ámbito de estudio}

Las reuniones del grupo de expertos se llevaron a cabo en Valencia en el Muy Ilustre Colegio Oficial de Farmacéuticos (MICOF) durante un periodo de 18 meses, desde abril de 2016 hasta septiembre de 2017. 

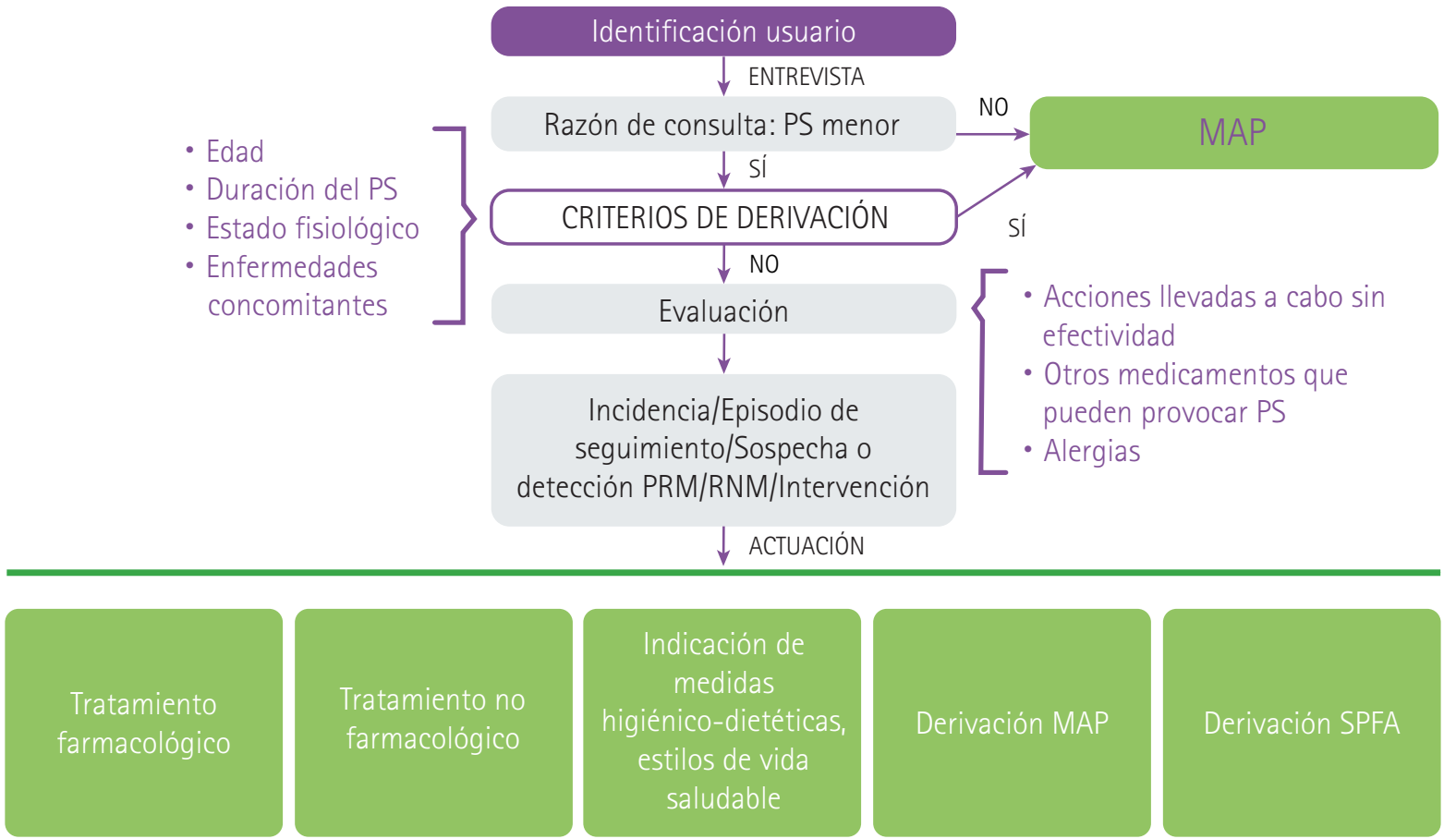

\section{Registro y evaluación}

PS: Problema de salud, MAP: Médico de Atención Primaria, RNM: Resultado Negativo de la Medicación, PRM: Problema Relacionado con la Medicación, SPFA: Servicios Profesionales Farmacéuticos Asistenciales

Figura 1 Diagrama de procedimiento del Servicio de Indicación Farmacéutica (Foro AF-FC) (3)

\section{Participantes}

Para la formación del grupo de expertos se consideraron farmacéuticos comunitarios y médicos de atención primaria de cuatro asociaciones diferentes: SEFAC (Sociedad Española de Farmacia Familiar y Comunitaria) y del MICOF (Muy Ilustre Colegio de Farmacéuticos de Valencia), semFYC (Sociedad Española de Medicina de Familia y Comunitaria) y SEMERGEN (Sociedad Española de Médicos de Atención Primaria). Cada asociación seleccionó a dos profesionales sanitarios especializados en el tema de los síntomas menores. El grupo de expertos fue liderado por un moderador del Grupo de Investigación en Atención Farmacéutica de la Universidad de Granada (GIAF-UGR).

\section{Procedimiento}

Se llevó a cabo una primera reunión con el objetivo de organizar las funciones del grupo, determinar la función de cada uno de los miembros, el método de obtención de datos, orga- nización del calendario de trabajo, reuniones necesarias para el consenso y estructura de los resultados obtenidos mediante revisión de la bibliografía.

Se revisaron los sintomas menores ya incluidos en el documento previo de 2008 (6) y se seleccionaron los síntomas menores más prevalentes no contemplados para su inclusión en la actualización. Se organizaron cuatro parejas formadas por un médico y un farmacéutico para la revisión de la bibliografía y se dividieron los síntomas considerados en los diferentes subgrupos de trabajo.

Se revisó la estructura para los diferentes sintomas menores que incluían originalmente los apartados: puntos clave indispensables a conocer por el farmacéutico (concepto del sintoma menor, causas más frecuentes que lo producen, medicamentos que pueden ocasionar síntomas que se confundan con el síntoma menor, criterios de derivación al médico, tratamiento no farmacológico y farmacológico mono componente que no requiera prescripción médica).
Para llevar a cabo la revisión de la bibliografía se consideró desde el 01/01/2007 (fecha final de revisión del documento inicial) hasta el 31/12/2016 utilizando las siguientes fuentes: Cochrane library, BMJ Clinical evidence, MEDLINE, Guía de Actuación en Atención Primaria de semFYC, Guía completa de consulta farmacoterapéutica Martindale, Bot Plus 2.0 del Consejo General de Colegios Oficiales de Farmacéuticos, National Guideline Clearinghouse, Scottish Intercollegiate Guidelines Network (SIGN), National Institute for Health and Care Excellence (NICE) y UpToDate.

En las reuniones posteriores del grupo de expertos se pusieron en común las conclusiones obtenidas en la búsqueda bibliográfica y se consensuó el trabajo realizado por cada pareja de expertos. Las reuniones fueron facilitadas por el moderador y audio grabadas mediante consentimiento previo para la elaboración de un documento con las conclusiones de cada reunión. 


\section{Resultados}

Se realizaron nueve reuniones del grupo de expertos para el consenso del documento. La guía elaborada (7) iba dirigida al farmacéutico comunitario. Se consensuaron protocolos normalizados de trabajo para 31 síntomas menores diferentes distribuidos en cinco grupos. El documento inicial incluía 27 sintomas menores $\mathrm{y}$, puesto que dos sintomas como eran sindrome catarral y gripal se agruparon en uno, se añadieron cinco síntomas nuevos (flatulencia, hiperhidrosis, pie de atleta, estrés, e irritación ocular que se dividió en ojo rojo y ojo seco). Además, lesiones eccematosas se sustituyó por dermatitis y dolor de espalda por dolor articular y de espalda. Los cinco grupos finales de síntomas se incluyen en la tabla 1.

Respecto a la estructura considerada para cada uno de los síntomas, se amplió la información considerada en cada caso para contemplar la información incluida en la tabla 2.

En el apartado, causas más frecuentes, se incluyó un listado de medicamentos que podian causar el sintoma menor. En general, los medicamentos no son la causa más frecuente, sin embargo, desde la perspectiva farmacéutica, este tipo de información resulta de gran utilidad práctica, incluso para otro tipo de servicios, como es el caso del seguimiento farmacoterapéutico.

Además, se añadió un nuevo apartado al final de "Principios activos" que consideraba todos principios activos incluidos, así como indicaciones y dosis autorizadas de los medicamentos para dispensar sin receta médica, las desventajas de su uso (efectos adversos muy frecuentes), las contraindicaciones absolutas e interacciones de relevancia clínica, y la información para un uso correcto por parte del paciente. Se incluyeron tanto tratamientos con un único principio activo como aquellos en asociación.

Para la elaboración de la guía se tuvieron en cuenta tanto la población diana (aquellas personas que acudieran a la farmacia consultando por un síntoma menor o demandando un medicamento para un problema de salud autodiagnosticado), como los profesionales sanitarios que podrían hacer uso de ésta (farmacéuticos comunitarios). La redacción y presentación de la guía se realizó de forma

Tabla 1 Sintomas menores incluidos en la guía de sintomas menores

\begin{tabular}{|l|l|}
\hline \multicolumn{1}{|c|}{ Grupo } & \multicolumn{1}{|c|}{ Sintoma menor } \\
\hline Respiratorios & Congestión nasal \\
& Sindrome catarral y gripal \\
& Tos \\
\hline Dolor moderado & Cefalea \\
& Dolor articular y de espalda \\
& Dolor dentario \\
& Dolor de garganta \\
& Dolor menstrual o dismenorrea primaria \\
\hline Digestivos & Acidez o pirosis \\
& Aerofagia, flatulencia y meteorismo \\
& Diarrea aguda \\
& Estreñimiento \\
Vómitos \\
\hline Dermatológicos & Acné \\
& Aftas (úlceras bucales) \\
Dermatitis \\
Heridas cutáneas \\
\hline Herpes labial \\
Hiperhidrosis \\
Picaduras \\
Pie de atleta \\
Ouemaduras cutáneas \\
Urticaria \\
Estrés \\
Fiebre \\
Hemorroides \\
Insomnio \\
Ojo rojo \\
Ojo seco \\
Vaginitis \\
\hline
\end{tabular}

ordenada, clara y práctica, a modo de cuaderno para facilitar su utilización durante la prestación de SIF en la farmacia comunitaria. En el Anexo 1 se incluye el primer síntoma menor de los protocolos a modo de ejemplo.

\section{Discusión}

Para conseguir los mejores resultados en salud de las personas es fundamental el trabajo coordinado y sinérgico de los profesionales sanitarios. Por lo tanto, para que el SIF sea seguro y efectivo, la actuación del farmacéutico debe de estar orientada por criterios científicos y técnicos consensuados entre médicos y farmacéuticos. Con este propósito, la guía elaborada recoge los aportes y criterios consensuados para contribuir a la mejora continua de la actuación del farmacéutico en el manejo de sínto- mas menores en pacientes sin riesgo, con énfasis en la definición de indicadores que determinen la necesidad de derivar el paciente al médico de atención primaria.

Cuando un paciente tiene un problema de salud, decide en función de su autonomía qué va a hacer para tratar de resolverlo (automedicación o acudir a un profesional sanitario). En muchas ocasiones, especialmente cuando percibe el problema de salud como banal, acude al farmacéutico para que éste decida sobre la solución más adecuada a su problema. En este contexto, los objetivos del farmacéutico son, en primer lugar, valorar si el problema de salud por el que consulta el paciente es un síntoma o trastorno banal (valoración del riesgo); en segundo lugar, indicar al paciente la opción más adecuada para resolver su problema de salud, incluyendo la 
Tabla 2 Estructura utilizada en la descripción de cada sintoma menor

\begin{tabular}{|c|c|}
\hline Apartado & Descripción \\
\hline Concepto & Describe el síntoma menor, diferenciándolo de otros procesos no banales. \\
\hline Causas más frecuentes & $\begin{array}{l}\text { Motivos que dan lugar a la aparición del sintoma, incluyendo medicamentos que pueden } \\
\text { causarlo. }\end{array}$ \\
\hline \multirow{5}{*}{$\begin{array}{l}\text { Criterios de derivación al } \\
\text { médico }\end{array}$} & Edad. \\
\hline & $\begin{array}{l}\text { Sintomas de alarma: otros sintomas o signos que, cuando los manifiesta o se reconocen en el } \\
\text { paciente, señalan la necesidad de la valoración por parte del médico. }\end{array}$ \\
\hline & $\begin{array}{l}\text { Duración de los sintomas: tiempo crítico de evolución del sintoma tras el cual el farmacéutico } \\
\text { puede sospechar que no se trata de un síntoma menor. }\end{array}$ \\
\hline & Otros problemas de salud y/o medicamentos. \\
\hline & Situaciones especiales. \\
\hline \multirow{3}{*}{$\begin{array}{l}\text { Recomendaciones para la } \\
\text { prevención y tratamiento }\end{array}$} & Prevención: medidas para evitar la aparición del síntoma menor. \\
\hline & $\begin{array}{l}\text { Tratamiento no farmacológico: medidas para tratar el sintoma menor sin incluir los } \\
\text { medicamentos. Considera recomendaciones higiénicas, nutricionales, cambio del estilo de vida, } \\
\text { etc. }\end{array}$ \\
\hline & $\begin{array}{l}\text { Tratamiento farmacológico: principios activos incluidos en medicamentos de publicidad } \\
\text { al público (sin receta médica) con indicación para los sintomas menores y con probada } \\
\text { efectividad y seguridad en el tratamiento de cada sintoma según la evidencia disponible. }\end{array}$ \\
\hline Bibliografia consultada & Fuentes bibliográficas utilizadas en la elaboración del protocolo de dicho sintoma menor. \\
\hline
\end{tabular}

derivación al médico (paciente con riesgo), la indicación de un medicamento sin necesidad de prescripción médica o de medidas no farmacológicas (paciente sin riesgo). Por último, proporcionar la información necesaria al paciente y protegerlo frente a la posible aparición de problemas de salud asociados a la medicación, detectando y corrigiendo sus causas prevenibles. Los criterios de derivación son indispensables, ya que el uso de medicamentos publicitarios puede retrasar la consulta del paciente al médico por problemas de salud complejos. Además, un elevado número de médicos desconocen el uso que hacen los pacientes de medicamentos publicitarios, lo que puede provocar que haya problemas de salud que no sean diagnosticados o problemas de salud no controlados por el mal uso de dichos medicamentos publicitarios.

Las derivaciones al médico de atención primaria o servicio de urgencias realizadas durante el SIF son fundamentales para garantizar la seguridad del paciente detectando aquellos casos no banales que preci- san de diagnóstico médico. En un estudio llevado a cabo en España en el año 2003 (8), se identificó que el farmacéutico realizó derivaciones en el $19,4 \%$ de las consultas del servicio, en el 5,2\% se dispensó algún medicamento o producto de parafarmacia previamente. En otro estudio posterior publicado en 2011 (9) se identificó que esta cifra tan solo ascendió al 2,4\%, aunque se estimó además que casi en el $10 \%$ de las consultas se les recomendó un medicamento y seguidamente fueron derivados al médico. Estas cifras están lejos del dato aportado en el último estudio realizado a nivel nacional por la Sociedad Española de Farmacia Familiar y Comunitaria (SEFAC) en 2015 (10), en el que se identificó una derivación del $29 \%$ de los pacientes; este estudio además indicaba que tan solo en el $7 \%$ (11) de las consultas del SIF cuya intervención era la derivación al médico, el farmacéutico generaba un informe para el paciente. Todo ello pone de manifiesto la variabilidad existente en la derivación a otros profesionales sanitarios dentro del SIF y la necesi- dad de protocolos consensuados con médicos para su disminución.

Además, en los casos en los que el farmacéutico considere que el síntoma menor requiere de un medicamento, la elección del principio activo debe estar basada en la mejor evidencia científica disponible. Por ello, el cumplimiento de esta actividad por parte del farmacéutico, requiere de conocimientos actualizados sobre los principios activos disponibles sin receta médica, especialmente sobre su efectividad y seguridad en el tratamiento de los trastornos menores. Además de la selección del medicamento más adecuado para el paciente, el farmacéutico debe proporcionar una información mínima sobre el objetivo del tratamiento, la pauta, la posología, la duración del mismo y qué hacer en caso de no mejoría o empeoramiento.

El servicio implica un buen uso de los medicamentos, pues todos ellos, tanto de prescripción como publicitarios, deben saber manejarse adecuadamente y utilizarse solo cuando realmente sean necesarios (muchos síntomas menores no precisan tratamiento 
farmacológico). La presencia del farmacéutico es una garantía para alcanzar este objetivo. Además, esto también puede ayudar a una automedicación responsable por parte de la población, evitando el consumo de medicamentos en las situaciones en las que no estén indicados y puedan poner en riesgo la salud del paciente.

La principal limitación de este estudio se basó en el hecho de que no fuese una revisión sistemática de la bibliografía, una mejora que podría realizarse en el futuro, así como una evaluación externa de dicha guía mediante el instrumento AGREE (12), con el fin de evaluar la calidad y el grado de recomendación.

El paso siguiente tras la elaboración de esta guía debía ser la evaluación de su impacto clínico y humanístico en el paciente. Para ello se puso en marcha el programa INDI$C A+P R O$ (5).

\section{Conclusión}

El documento desarrollado mediante colaboración entre profesionales de la medicina y la farmacia es una herramienta que contribuye a la mejora de la actuación del farmacéutico en el manejo de los síntomas menores desde la farmacia comunitaria, con énfasis en la definición de indicadores que determinan la necesidad de derivar el paciente al médico de atención primaria. Con esta guía se pretende mejorar la labor asistencial del farmacéutico comunitario reforzando el SIF.

\section{Referencias bibliográficas}

1. Faus Dáder, MJ, Amariles Muñoz P, Martinez-Martinez $\mathrm{F}$ et al Atención farmacéutica servicios farmacéuticos orientados al paciente. ISBN: 978-8416992-59-1.

2. Foro de Atención Farmacéutica en Farmacia Comunitaria. Sexto comunicado Foro AF-FC, SERVICIOS PROFESIONALES FARMACÉUTICOS ASISTENCIALES. Madrid: 2016. Disponible en: https://www.portalfarma. com/inicio/serviciosprofesionales/forofarmaciacomunitaria/comunicaciones/Documents/2016-6o-Comunicado-FOR0-AF-FC-Servicios-Profesionales-Farmaceuticos-Asistenciales. pdf

3. Foro de Atención Farmacéutica en Farmacia Comunitaria, panel de expertos. Guía práctica para los Servicios Profesionales Farmacéuticos Asistenciales en la Farmacia Comunitaria. Madrid: Medea; 2019.

4. Organización Mundial de la Salud (OMS). El papel del farmacéutico en el autocuidado y la automedicación. Reporte de la $4^{\mathrm{a}}$ Reunión del Grupo Consultivo de la OMS sobre el papel del farmacéutico. La Haya: Organización Mundical de la Salud; 1998.

5. Amador-Fernández N, Baixauli-Fernández VJ, Climent-Catalá MT et al. INDICA+PRO, informe sobre la evaluación del impacto clínico, humanístico y económico del servicio de indicación farmacéutica en el ámbito de la farmacia comunitaria. Granada: Grupo de Investigación en Atención Farmacéutica; 2019.

6. Baos V, Faus Dáder MJ et al. Protocolos de Indicación Farmacéutica y
Criterios de Derivación al Médico en Síntomas Menores. Granada: Grupo de Investigación en Atención Farmacéutica de la Universidad de Granada; 2008.

7. Faus Dáder, MJ, Gómez Martínez JC, Martínez Martínez F et al. Protocolos de Indicación Farmacéutica y Criterios de Derivación al Médico en Síntomas Menores. Granada: Editorial Técnica Avicam; 2018.

8. Baixauli-Fernández VJ, Barbero-González A, Salar-Ibáñez L. Las consultas de indicación farmacéutica en la Farmacia Comunitaria. Pharm Care Esp. 2005;7(2):54-61.

9. Ocaña A. Efectividad del proceso estructurado de asesoramiento en síntomas menores frente al asesoramiento habitual en Farmacias Comunitarias españolas [Tesis Doctoral]. Granada: Universidad de Granada, 2011.

10. Salar Ibáñez L, Espejo Guerrero J, Gómez Martínez J, Prats Más R, Eyaralar Riera T, Barbero González A. Criterios de derivación a un servicio médico en 'I-VALOR'. Farmacéuticos Comunitarios. 2016; 8(Suplemento 1).

11. González Hernández P, Moreno Fernández $\mathrm{P}$, Gómez Martínez JC, Salar Ibáñez L, Prats Mas R, Barbero González A, Espejo Guerrero J, Eyaralar Riera T. I-valor: Indicación Farmacéutica en erosiones cutáneas. Farmacéuticos Comunitarios. 2016; 8(Suplemento 1).

12. Development and validation of an international appraisal instrument for assessing the quality of clinical practice guidelines: the AGREE project. Qual Saf Health Care. 2003 Feb;12(1):18-23. doi:10.1136/ qhc. 12.1 .18 


\section{Anexo 1. Protocolo consensuado para el síntoma menor "Congestión nasal" (7)}

\section{CONGESTIÓN NASAL}

\section{CONCEPTO}

Sensación de dificultad al paso del aire a través de las fosas nasales. Fundamentalmente, se produce por la vasodilatación de los vasos sanguíneos de la mucosa nasal. Suele ser un síntoma de infección de vías respiratorias superiores o signo de alguna enfermedad aguda o crónica, pudiendo afectar a una o ambas fosas nasales.

Por su parte, el término "rinorrea" hace referencia a la eliminación de mucosidad, de densidad variable, a través de las fosas nasales.

\section{CAUSAS MÁS FRECUENTES}

- Infección de vías respiratorias superiores (causa más frecuente).

- Rinitis infecciosas de tipo viral (catarro común), es la causa más común de rinitis (inflamación de la mucosa nasal).

- Rinitis alérgica, suele ser estacional y acompañada de estornudos (provocada por ácaros, polen, etc).

- Rinitis relacionada con alteración endocrina: Hipotiroidismo, embarazo, menopausia.

- Sinusitis aguda y crónica.

- Pólipos nasales.

- Cuerpo extraño nasal (suele ocurrir en niños y se presenta acompañado de rinorrea purulenta unilateral).

- Rinitis vasomotora: Predomina la obstrucción y la rinorrea con secreción transparente.

- Rinitis secundaria a cocaína, alcohol o a nicotina.

- Patología del tabique nasal (desviación del tabique). 
PROTOCOLOS DE INDICACIÓN FARMACÉUtICA

y CRiterios de Derivación al médico en Síntomas Menores

- Hipertrofia adenoidea (obstrucción persistente en niños).

- Rinitis farmacológica o medicamentosa. Se origina principalmente por un uso inadecuado de descongestivos nasales.

- Vasoconstrictores tópicos: Estimulan los receptores alfa adrenérgicos, produciendo vasoconstricción y, por tanto, disminuyendo la hinchazón de la mucosa. Con el uso repetido o prolongado se produce una congestión de rebote que conduce al paciente a aumentar la dosis y frecuencia del uso del vasoconstrictor. El mecanismo responsable puede deberse a una desensibilización de los receptores adrenérgicos o a una disminución de la noradrenalina endógena.

Otros medicamentos que pueden causar congestión nasal:

- Analgésicos antiinflamatorios no esteroideos (AINE): Ácido acetilsalicílico, ácido mefenámico, nimesulida.

- Antihipertensivos: Inhibidores de la enzima conversora de angiotensina (IECA), bloqueantes beta, hidroclorotiazida, reserpina, amilorida, doxazosina, prazosina, hidralazina, metildopa, clonidina, guanetidina, fentolamina.

- Inhibidores de la 5- Fosfodiesterasa: Sildenafilo, tadalafilo, vardenafilo.

- Antipsicóticos: Clorpromazina, risperidona, tioridazina.

- Clordiazepóxido-amitriptilina.

- Anticonceptivos orales.

\section{CRITERIOS DE DERIVACIÓN AL MÉDICO}

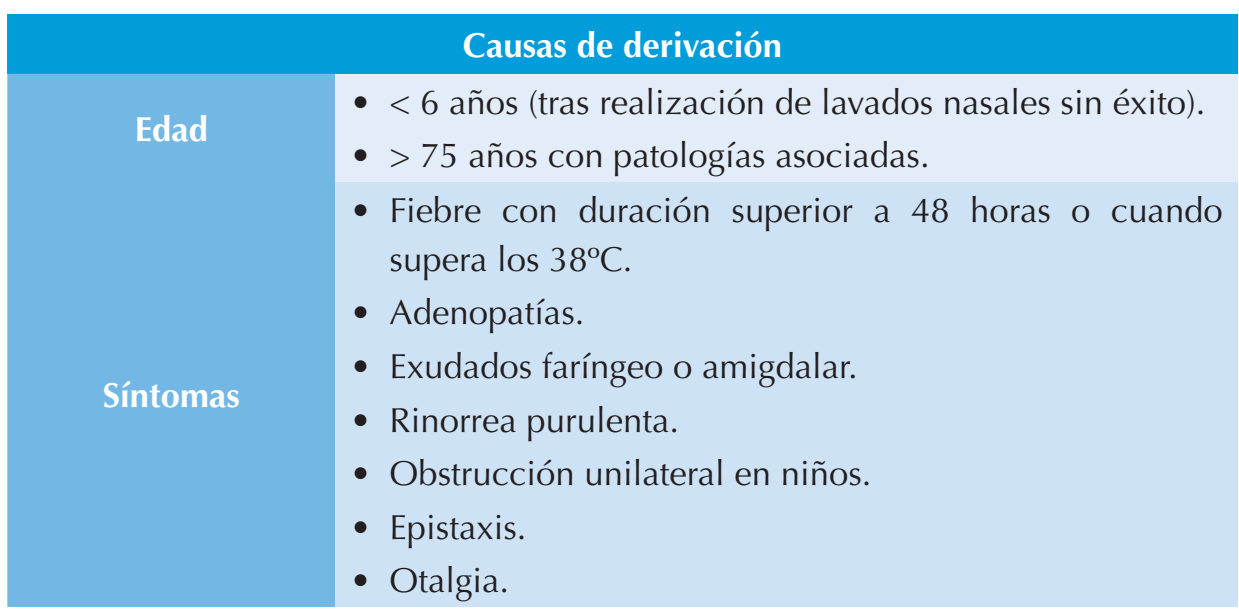




\section{Duración de los síntomas}

Enfermedad $y / 0$ medicamentos

Situaciones especiales
- > 3 días con tratamiento sin mejoría.

- $>2$ semanas sin tratamiento.

- Rinitis medicamentosa.

- Enfermedad cardíaca.

- Enfermedad respiratoria mal controlada (asma/EPOC).

- Uso de antidepresivos tricíclicos e IMAO.

- Embarazo y lactancia (tras realización de lavados nasales sin éxito).

- Inmunosupresión.

\section{RECOMENDACIONES PARA LA PREVENCIÓN Y EL TRATAMIENTO}

\section{Prevención y tratamiento no farmacológico}

Es esencial tanto para la prevención como para el tratamiento no farmacológico evitar los factores desencadenantes, ya sean irritantes, alérgenos o fármacos. También es importante llevar a cabo las siguientes medidas higiénicodietéticas:

- Evitar los cambios bruscos de temperatura, tabaco o alcohol, ya que producen mayor sequedad de las mucosas.

- Realizar lavados nasales con soluciones salinas fisiológicas o preparados con agua de mar isotónica, que ayudan a eliminar la mucosidad y descongestionan.

- Mantener el ambiente húmedo, es recomendable no permanecer en lugares cerrados o mal ventilados.

- Mantener una ingesta de líquidos apropiada para prevenir la sequedad de las mucosas de las vías respiratorias.

- Realizar inhalaciones de vapor, que ayudan a fluidificar y a expulsar la mucosidad.

- Si esto no fuera suficiente, para un cuadro auto-limitado, se justifica el uso de tratamiento farmacológico durante el periodo más breve de tiempo posible. 
PROTOCOLOS DE INDICACIÓN FARMACÉUTICA

y CRITERIOS de Derivación al médico en Síntomas Menores

\section{Tratamiento farmacológico}

Descongestionantes adrenérgicos: Los descongestionantes reducen la congestión nasal. Se usan normalmente en forma de aerosoles o gotas nasales. Están contraindicados en personas con arritmia, hipertensión, hipertiroidismo, enfermedad coronaria, glaucoma, retención urinaria y patología psiquiátrica. No deben emplearse nunca en bebés y niños pequeños. Su uso continuado puede producir un efecto rebote (rinitis medicamentosa) por lo que no deben usarse de manera continua más de 3 días.

Antihistamínicos: Eficaces en el tratamiento de los síntomas leves/ moderados de la rinitis alérgica como estornudos, prurito o rinorrea. Los de segunda generación producen menos sedación y trastornos psicomotores y tienen mayor duración de acción.

Los principios activos indicados son:

- Antihistamínicos: Cetirizina (p. 226), Ciproheptadina (p. 227), Ebastina (p. 229), Loratadina (p. 229).

- Corticoides: Propionato de fluticasona (p. 238).

- Descongestionantes adrenérgicos tópicos: Fenilefrina (p. 260), Nafazolina (p. 261), Oximetazolina (p. 261), Tramazolina (p. 262), Xilometazolina (p. 262).

- Combinaciones:

- Pseudoefedrina/Cetirizina (p. 232).

- Pseudoefedrina/Ebastina (p. 233).

- Pseudoefedrina/Loratadina (p. 233).

- Oximetazolina/Clorfenamina (p. 263).

- Tramazolina/Clorfenamina (p. 263).

- Xilometazolina/Bromuro de Ipratropio (p. 264).

\section{BIBLIOGRAFÍA CONSULTADA}

Agencia Española del Medicamento y Productos Sanitarios. Centro de Información online de Medicamentos de la AEMPS [acceso 31 enero 2018]. Disponible en: https://www.aemps.gob.es/cima

Andrés Rodríguez NF, Botana Rey A, Crespo Suárez M, Iglesias L, Pouso Alcalde C, Rey F, Rivas M. Gripe y resfriado. En: Andrés Rodríguez NF, Fornos Pérez JA, Mera Gallego R, Barreiro Juncal M, Vérez Cotelo N, Prunell Hombre M, et 
al [coordinadores]. Guía Para el Servicio de Indicación Farmacéutica. Vigo: Cofano; 2016. Págs. 175-188. ISBN: 978-84-617-5149-5.

Consejo General de Colegios Oficiales de Farmacéuticos. Bot Plus. Base de Datos del Conocimiento Sanitario. Madrid: CGCOF; 2016.

Grupo de trabajo de El Farmacéutico. Gripe y resfriado. El farmacéutico; 2015 [acceso 31 enero 2018]. Disponible en: http://elfarmaceutico.es/index.php/la-revista/ itemlist/category/476-numero-526-1-octubre-2015

Kahan S, Smith EG. Signos y síntomas en una página. Barcelona: Lippincott Williams \& Wilkins Mayo; 2007.

Martín Mateos AJ, Romero Sánchez E, De Mier Morales M. Rinitis. Fisterra; 2011 [acceso 31 enero 2018]. Disponible en: http://www.fisterra.com/guias-clinicas/ rinitis/ 